\title{
ESTIMATION OF WEIBULL PARAMETERS USING A RANDOMIZED NEIGHBORHOOD SEARCH FOR THE SEVERITY OF FIRE ACCIDENTS
}

\author{
${ }^{1}$ Soontorn Boonta, ${ }^{2}$ Anchalee Sattayatham and ${ }^{1}$ Pairote Sattayatham \\ ${ }^{1}$ Institute of Science, School of Mathematics, \\ Suranaree University of Technology, NakhonRatchasima 30000, Thailand \\ ${ }^{2}$ Faculty of Liberal Arts, Mahidol University, Bangkok 10400, Thailand
}

Received 2012-12-19; Revised 2013-02-16; Accepted 2013-03-15

\begin{abstract}
In this study, we applied Randomized Neighborhood Search (RNS) to estimate the Weibull parameters to determine the severity of fire accidents; the data were provided by the Thai Reinsurance Public Co., Ltd. We compared this technique with other frequently-used techniques: the Maximum Likelihood Estimator (MLE), the Method of Moments (MOM), the Least Squares Method (LSM) and the weighted least squares method (WLSM) and found that RNS estimates the parameters more accurately than do MLE, MOM, LSM or WLSM.
\end{abstract}

Keywords: Weibull Distribution, Parameter Estimation, Randomized Neighborhood Search

\section{INTRODUCTION}

The problem of estimating parameters in actuarial science is an important issue. Choosing an appropriate estimator is very important. In practice, constructive methods for parameter estimation are needed. The Maximum Likelihood Estimator (MLE), the Method of Moments (MOM), the Least Squares Method (LSM) and the Weighted Least Squares Method (WLSM) are frequently used for parameter estimation. Here, we consider the problem of the estimation of Weibull parameters. Many authors have investigated various aspects of this problem. Seyit and Ali (2009) presented power density method for Weibull parameters estimation. El-Mezouar (2010) proposed the Coefficient of Variation (CV) estimator comparing with Cran (1988) of the estimation of Weibull parameters. Yeliz et al. (2011) compared the method based on quantiles, maximum spacing method, MLE, MOM, LSM and WLSM for Weibull parameters estimation.

In this study, we propose the Randomized Neighborhood Search technique (RNS) for the Corresponding author: Soontorn Boonta, Institute of Science, School of Mathematics, Suranaree University of Technology, NakhonRatchasima 30000, Thailand estimation of the Weibull parameters for the claim severity of fire accidents; the data were provided by the Thai Reinsurance Public Co., Ltd. Five estimation methods (MLE, MOM, LSM, WLSM and RNS) were used to estimate the Weibull parameters. Based on chisquared value, RNS estimates the parameters more accurately than do MLE, MOM, LSM or WLSM.

\section{MATERIALS AND METHODS}

\subsection{Weibull Distribution}

Catastrophe insurance covers large insurance losses that happen infrequently, but have payouts for claims. Examples include large-scale fire, windstorm or flood insurance. In case of catastrophes, claim severity has heavy tails. The Weibull distribution with a shape parameter of less than one and a scale parameter greater than zero is a clear example of heavy-tailed distribution. The probability density and cumulative distribution function forth three-parameter Weibull random variable $\mathrm{X}$, in which each is defined by Equation 1 and 2: 
$f(x ; \alpha, \beta, \gamma)=\frac{\alpha}{\beta}\left(\frac{x-\gamma}{\beta}\right)^{\alpha-1} \exp \left(-\left(\frac{x-\gamma}{\beta}\right)^{\alpha}\right)$

And:

$$
\mathrm{F}(\mathrm{x} ; \alpha, \beta, \gamma)=1-\exp \left(-\left(\frac{\mathrm{x}-\gamma}{\beta}\right)^{\alpha}\right)
$$

where, $\alpha>0, \beta>0$ and $\gamma>0$ and are the shape, scale and location parameters respectively. In this study, we consider claim severity $\mathrm{x}$ with a cost greater than 20 million baht. Thus we set $\gamma=20$ Let $y=x-\gamma$. It then follows from (1) and (2) that for each $\mathrm{y} \geq 0$ :

$$
f(y ; \alpha, \beta)=\frac{\alpha}{\beta}\left(\frac{y}{\beta}\right)^{\alpha-1} \exp \left(-\left(\frac{y}{\beta}\right)^{\alpha}\right)
$$

And Equation 3:

$$
\mathrm{F}(\mathrm{y} ; \alpha, \beta)=1-\exp \left(-\left(\frac{\mathrm{y}}{\beta}\right)^{\alpha}\right)
$$

\subsection{Estimation of the Weibull Parameters}

\subsubsection{Maximum Likelihood Estimator (MLE)}

Let $\mathrm{y}_{1}, \mathrm{y}_{2}, \ldots, \mathrm{y}_{\mathrm{n}}$ be a random sample for the Weibull distribution, then the likelihood function $\mathrm{L}$ is defined as Equation 4:

$$
\mathrm{L}\left(\mathrm{y}_{1}, \mathrm{y}_{2}, \ldots, \mathrm{y}_{\mathrm{n}} ; \alpha, \beta\right)=\prod_{\mathrm{i}=1}^{\mathrm{n}} \frac{\alpha}{\beta}\left(\frac{\mathrm{y}_{\mathrm{i}}}{\beta}\right)^{\alpha-1} \exp \left(-\left(\frac{\mathrm{y}_{\mathrm{i}}}{\beta}\right)^{\alpha}\right)
$$

On taking the logarithms of (4), differentiated with respect to $\beta$ and $\alpha$ and equal to zero, one gets:

$$
\begin{aligned}
& \frac{\partial \ln \mathrm{L}}{\partial \beta}=-\frac{\mathrm{n}}{\beta}+\frac{1}{\beta^{\alpha+1}} \sum_{\mathrm{i}=1}^{\mathrm{n}}\left(\mathrm{y}_{\mathrm{i}}\right)^{\alpha}=0, \\
& \frac{\partial \ln \mathrm{L}}{\partial \alpha}=\frac{\mathrm{n}}{\alpha}-\mathrm{n} \ln \beta+\sum_{\mathrm{i}=1}^{\mathrm{n}} \ln \mathrm{y}_{\mathrm{i}}-\sum_{\mathrm{i}=1}^{\mathrm{n}}\left(\frac{\mathrm{y}_{\mathrm{i}}}{\beta}\right)^{\alpha} \ln \left(\frac{\mathrm{y}_{\mathrm{i}}}{\beta}\right)=0
\end{aligned}
$$

After solving the above two equations, we obtain Equation 5 and 6:

$$
\beta=\left(\frac{1}{n} \sum_{i=1}^{n} y_{i}^{\alpha}\right)^{\frac{1}{\alpha}}
$$

$$
\alpha=\left[\frac{\sum_{\mathrm{i}=1}^{\mathrm{n}}\left(\mathrm{y}_{\mathrm{i}}\right)^{\alpha} \ln \mathrm{y}_{\mathrm{i}}}{\sum_{\mathrm{i}=1}^{\mathrm{n}}\left(\mathrm{y}_{\mathrm{i}}\right)^{\alpha}}-\frac{1}{\mathrm{n}} \sum_{\mathrm{i}=1}^{\mathrm{n}} \ln \mathrm{y}_{\mathrm{i}}\right]^{-1}
$$

The value $\alpha$ has to be obtained from (6) by NewtonRaphson and then $\alpha$ is inserted into (5) to obtain $\beta$.

\subsection{Methods of Moments (MOM)}

We know that the kth moment $\mu_{\mathrm{k}}$ for the Weibull distribution is given by:

$$
\mu_{\mathrm{k}}=\beta^{\mathrm{k}} \Gamma\left(1+\frac{\mathrm{k}}{\alpha}\right)
$$

where, $\Gamma(\mathrm{t})$ defines the gamma function as:

$$
\Gamma(t) \int_{0}^{\infty} e^{-x} x^{t-1} d x, t>0
$$

In particular, the mean $\mu$ (the first moment) and the variance $\sigma^{2}$ are Equation 7 and 8:

$$
\mu=\beta \Gamma\left(1+\frac{1}{\alpha}\right)
$$

$$
\sigma^{2}=\mu_{2}-(\mu)^{2}=\beta^{2}\left[\Gamma\left(1+\frac{2}{\alpha}\right)-\Gamma^{2}\left(1+\frac{1}{\alpha}\right)\right]
$$

The coefficient of variation $\mathrm{CV}$ for the Weibull distribution can be determined as follows Equation 9:

$\mathrm{CV}=\frac{\sigma}{\mu}=\frac{\sqrt{\Gamma\left(1+\frac{2}{\alpha}\right)-\Gamma^{2}\left(1+\frac{1}{\alpha}\right)}}{\Gamma\left(1+\frac{1}{\alpha}\right)}$

The shape parameter $\alpha$ as appears in (9) will be determined by bisection and the scale $\beta$ may be calculated from (7).

Another method of moment has been proposed by Cran (1988). Let $\left.x_{(1)} \leq x_{(2)} \leq \ldots\right) \leq x_{(n)}$ be an ordered random sample of the cumulative distribution function $\mathrm{F}_{(\mathrm{y})}$ as in (3). Then $F_{(y)}$ can be estimated by $S_{n}(x)$ where: 


$$
S_{n}(x)= \begin{cases}0, & x<x_{(1)}, \\ \frac{r}{n}, & x_{(r)} \leq x<x_{(r+1)}, r=1, \ldots, n-1 \\ 1, & x_{(n)} \leq x .\end{cases}
$$

Then the population moment $\mu_{\mathrm{k}}$ is estimated by:

$$
\begin{aligned}
\mathrm{m}_{\mathrm{k}} & =\int_{0}^{\infty}\left[1-\mathrm{S}_{\mathrm{n}}(\mathrm{x})\right]^{\mathrm{k}} \mathrm{dx} \\
& =\sum_{\mathrm{r}=0}^{\mathrm{n}-1}\left(1-\frac{\mathrm{r}}{\mathrm{n}}\right)^{\mathrm{k}}\left(\mathrm{x}_{(\mathrm{r}+1)}-\mathrm{x}_{(\mathrm{r})}\right), \mathrm{x}_{(0)}=0
\end{aligned}
$$

He expresses the parameters in terms of lower order moment as follows:

$$
\alpha=(\ln 2)\left(\ln \left(\mu_{1}-\mu_{2}\right)-\ln \left(\mu_{2}-\mu_{4}\right)\right)^{-1}
$$

And:

$$
\beta=\mu_{1}\left(\Gamma\left(1+\frac{1}{\alpha}\right)\right)^{-1}
$$

Therefore, $\alpha$ and $\beta$ can be obtained by substituting $\mathrm{m}_{1}, \mathrm{~m}_{2}$ and $\mathrm{m}_{4}$ for $\mu_{1}, \mu_{2}$ and $\mu_{4}$ respectively.

\subsection{Least Squares Method (LSM)}

We note from (3) that a probability $F_{i}$ is assigned to each $y_{i}$. Since true value of $F_{i}$ is unknown, a prescribed estimator must to be used. The following four expressions which are often used to define the probability estimator Equation 10a-10d.

$\mathrm{F}_{\mathrm{i}}=\frac{\mathrm{i}-0.5}{\mathrm{n}}$

$F_{i}=\frac{i}{n+1}$

$F_{i}=\frac{i-0.3}{n+0.4}$

$F_{i}=\frac{i-3 / 8}{n+1 / 4}$

where, $F_{i}$ is the probability for the ith ranked $y_{i}$ and $n$ is the sample size.
By applying the logarithm to (3), we get a linear form:

$\ln \ln \left[\frac{1}{1-\mathrm{F}}\right]=\alpha \ln \mathrm{y}-\alpha \ln \beta$

The shape parameter $\alpha$ can be obtained from the slope term in (11) and the scale parameter $\beta$ can be solved from the intercept term.

\subsection{Weighted Least Squares Method (WLSM)}

For this method, we follow the technique given by $\mathrm{Wu}$ et al. (2006). Equation (11) can be rewritten in the form $\mathrm{Y}=\mathrm{mS}+\mathrm{b}$, where:

$$
\mathrm{Y}=\ln \ln \left[\frac{1}{1-\mathrm{F}}\right], \mathrm{m}=\alpha, \mathrm{S}=\ln \mathrm{y} \text { and } \mathrm{b}=-\alpha \ln \beta
$$

WLSM is based on the hypothesis that a straight line fitting must minimize the weighted sum of the squares of deviations for the data $\mathrm{Y}_{\mathrm{i}}$ from the fitting function $\mathrm{Y}\left(\mathrm{S}_{\mathrm{i}}\right)$, so the equation:

$$
\mathrm{l}^{2}=\sum_{\mathrm{i}=1}^{\mathrm{n}} \mathrm{W}_{\mathrm{i}}\left(\mathrm{Y}_{\mathrm{i}}-\mathrm{b}-\mathrm{mS}_{\mathrm{i}}\right)^{2}
$$

gives the minimum value. By solving $\frac{\partial 1^{2}}{\partial \mathrm{m}}=\frac{\partial 1^{2}}{\partial \mathrm{b}}=0$, we compute:

$$
\begin{aligned}
& \mathrm{m}=\alpha=\frac{\sum_{\mathrm{i}=1}^{\mathrm{n}} \mathrm{W}_{\mathrm{i}} \sum_{\mathrm{i}=1}^{\mathrm{n}} \mathrm{S}_{\mathrm{i}} \mathrm{Y}_{\mathrm{i}} \mathrm{W}_{\mathrm{i}}-\sum_{\mathrm{i}=1}^{\mathrm{n}} \mathrm{S}_{\mathrm{i}} \mathrm{W}_{\mathrm{i}} \sum_{\mathrm{i}=1}^{\mathrm{n}} \mathrm{Y}_{\mathrm{i}} \mathrm{W}_{\mathrm{i}}}{\sum_{\mathrm{i}=1}^{\mathrm{n}} \mathrm{W}_{\mathrm{i}} \sum_{\mathrm{i}=1}^{\mathrm{n}} \mathrm{S}_{\mathrm{i}}^{2} \mathrm{~W}_{\mathrm{i}}-\left(\sum_{\mathrm{i}=1}^{\mathrm{n}} \mathrm{S}_{\mathrm{i}} \mathrm{W}_{\mathrm{i}}\right)^{2}}, \\
& \mathrm{~b}=\frac{\sum_{\mathrm{i}=1}^{\mathrm{n}} \mathrm{Y}_{\mathrm{i}} \mathrm{W}_{\mathrm{i}}-\alpha \sum_{\mathrm{i}=1}^{\mathrm{n}} \mathrm{S}_{\mathrm{i}} \mathrm{W}_{\mathrm{i}}}{\sum_{\mathrm{i}=1}^{\mathrm{n}} \mathrm{W}_{\mathrm{i}}}
\end{aligned}
$$

where, $\mathrm{W}_{\mathrm{i}}$ is the weight factor for the ith datum point. The parameter $\beta$ can be calculated from:

$$
\beta=\exp \left(-\frac{b}{m}\right)
$$

It is clear that LSM is a special case of WLSM at $\mathrm{W}_{\mathrm{i}}=1$. 
They used the weight factor based on the theory of error propagation Equation 12a and 12b:

$$
\begin{aligned}
& \mathrm{W}_{\mathrm{i}}=\left[\left(1-\mathrm{F}_{\mathrm{i}}\right) \ln \left(1-\mathrm{F}_{\mathrm{i}}\right)\right]^{2} \\
& \mathrm{~W}_{\mathrm{i}}=3.3 \mathrm{~F}_{\mathrm{i}}-27.5\left[1-\left(1-\mathrm{F}_{\mathrm{i}}\right)^{0.025}\right]
\end{aligned}
$$

Similar to LSM, the probability $F$ for each datum ranked in ascending order is also approximated by $\mathrm{F}_{\mathrm{i}}$ as shown from (10a) to (10d).

We consider a data set of fire insurance claims in Thailand from 2000 to 2004. These data were provided by the Thai Reinsurance Public Co., Ltd. They consist of the claim times and the claim severity $\mathrm{x}_{\mathrm{i}}$. The amount $\mathrm{y}_{\mathrm{i}}$ as shown in Table 1, is represents amounts above 20 million baht, i.e., $\mathrm{y}_{\mathrm{i}}=\mathrm{x}_{\mathrm{i}}-20$. For convenience, we still call the amount $y_{i}$ claim severity.

Table 2 shows the shape parameters $\alpha$ and scale parameters $\beta$ using different estimation methods for the data found in Table 1.

\subsection{Chi-Squared}

Chi-squared is defined as:

$$
x^{2}=\sum_{i=1}^{k} \frac{\left(O_{i}-E_{i}\right)^{2}}{E_{i}}
$$

where, $\mathrm{k}$ is the total number of intervals, $\mathrm{O}_{\mathrm{i}}$ is the observed frequency for intervali, $\mathrm{E}_{\mathrm{i}}$ is the expected frequency for interval i and:

$$
\mathrm{E}_{\mathrm{i}}=\mathrm{n}\left[\mathrm{F}\left(\mathrm{y}_{\mathrm{i}}\right)-\mathrm{F}\left(\mathrm{y}_{\mathrm{i}-1}\right)\right], \mathrm{i}=1,2, \ldots, \mathrm{F}\left(\mathrm{y}_{0}\right)=0
$$

Here $\mathrm{n}$ is the sample size, $\mathrm{F}$ is the cumulative distribution function as in (3) and $\mathrm{y}_{\mathrm{i}}, \mathrm{y}_{\mathrm{i}-1}$ are the endpoints of the interval.

We performed the chi-squared goodness of fit test for all methods in Table 2. The null hypothesis $\mathrm{H}_{0}$ : data is assumed for Weibull $(\alpha, \beta)$. We found that the chisquared value is less than the chi-squared critical value for degree of freedom 4 at a significance level of 0.05 For example, $\mathrm{H}_{0}$ : data is the assumed Weibull $(\alpha=$ $0.9286, \beta=30.0055)$. The chi-squared critical valuefor degree of freedom 4 at a significance level of 0.05 is 9.49, whereas the chi-squared value is 4.0569 (Table 3).

\begin{tabular}{|c|c|c|c|c|c|}
\hline \multicolumn{6}{|l|}{2000} \\
\hline 6-Mar & 12-Mar & 12-Mar & 25-Mar & 13-Jul & 26-Aug \\
\hline 15.5 & 6.4 & 44.9 & 107.3 & 37.7 & 1.8 \\
\hline 3-Sep & 24-Oct & & & & \\
\hline 47.3 & 28.5 & & & & \\
\hline \multicolumn{6}{|l|}{2001} \\
\hline 16-Jan & 28-Jan & 17-Feb & 22-Feb & 9-Mar & 19-Jun \\
\hline 3.6 & 2.3 & 64.6 & 1.4 & 31.5 & 0.7 \\
\hline 20-Jun & 5-Jul & 6-Aug & 24-Aug & 18-Sep & 23-Oct \\
\hline 20.1 & 9.3 & 6.7 & 12.4 & 56.5 & 13.2 \\
\hline 29-Nov & 1-Dec & & & & \\
\hline 5.7 & 40.2 & & & & \\
\hline \multicolumn{6}{|l|}{2002} \\
\hline 27-Jan & 2-Mar & 10-Apr & 13-Apr & 2-Jun & 23-Aug \\
\hline 112.2 & 0.9 & 45.8 & 35.3 & 13 & 2.1 \\
\hline 26-Oct & 29-Oct & & & & \\
\hline 4.2 & 24.4 & & & & \\
\hline \multicolumn{6}{|l|}{2003} \\
\hline 9-Jan & $5-\mathrm{Feb}$ & 8-Apr & 14-Apr & 7-May & 23-Nov \\
\hline 0.4 & 10.8 & 49.9 & 102.7 & 138.9 & 13.1 \\
\hline \multicolumn{6}{|l|}{2004} \\
\hline 2-Jan & 2-Jan & 2-Jan & 7-Feb & 28-Feb & 5-Mar \\
\hline 40 & 84.3 & 9.2 & 43.1 & 70 & 7.2 \\
\hline 14-Mar & 22-Apr & 8-Jul & 1-Nov & 24-Dec & \\
\hline 2.4 & 7.5 & 37.2 & 14.2 & 33.2 & \\
\hline
\end{tabular}
Thus we can assume that the distribution of the data (Table 1) is Weibull at a 5\% degree of significance.
Table 1. Claim times and claim severity $\mathrm{y}_{1}$ (million baht)

Table 2. Shape $\alpha$ and scale $\beta$ parametersusing various estimation methods

\begin{tabular}{llllll}
\hline Method & Type & $\mathrm{W}_{\mathrm{i}}$ & $\mathrm{F}_{\mathrm{i}}$ & $\alpha$ & $\beta$ \\
\hline 1 & MLE & - & - & 0.8633 & 28.8668 \\
2 & MOM (CV) & - & - & 0.9286 & 30.0055 \\
3 & MOM (Cran) & - & - & 0.9552 & 30.4239 \\
4 & LSM_1 & - & $10 \mathrm{a}$ & 0.8580 & 28.6168 \\
5 & LSM_2 & - & $10 \mathrm{~b}$ & 0.7984 & 29.1888 \\
6 & LSM_3 & - & $10 \mathrm{c}$ & 0.8310 & 28.8602 \\
7 & LSM_4 & - & $10 \mathrm{~d}$ & 0.8405 & 28.7721 \\
8 & WLSM_1 & $12 \mathrm{a}$ & $10 \mathrm{a}$ & 0.7647 & 29.9050 \\
9 & WLSM_2 & $12 \mathrm{a}$ & $10 \mathrm{~b}$ & 0.7455 & 30.1924 \\
10 & WLSM_3 & $12 \mathrm{a}$ & $10 \mathrm{c}$ & 0.7571 & 30.0176 \\
11 & WLSM_4 & $12 \mathrm{a}$ & $10 \mathrm{~d}$ & 0.7600 & 29.9750 \\
12 & WLSM_5 & $12 \mathrm{~b}$ & $10 \mathrm{a}$ & 0.7967 & 29.2036 \\
13 & WLSM_6 & $12 \mathrm{~b}$ & $10 \mathrm{~b}$ & 0.7710 & 29.5150 \\
14 & WLSM_7 & $12 \mathrm{~b}$ & $10 \mathrm{c}$ & 0.7868 & 29.3166 \\
15 & WLSM_8 & $12 \mathrm{~b}$ & $10 \mathrm{~d}$ & 0.7907 & 29.2713 \\
\hline
\end{tabular}

Table 3. Chi-Squared, $\alpha=0.9286$ and $\beta=30.0055$

\begin{tabular}{llllll}
\hline Row $^{\mathrm{i}}$ & $\mathrm{y}_{\mathrm{i}}$ & $\mathrm{F}\left(\mathrm{y}_{\mathrm{i}}\right)-\mathrm{F}\left(\mathrm{y}_{\mathrm{i}-1}\right)$ & $\mathrm{E}_{\mathrm{i}}$ & $\mathrm{O}_{\mathrm{i}}$ & $\left(\mathrm{O}_{\mathrm{i}}-\mathrm{E}_{\mathrm{i}}\right)^{2} / \mathrm{E}_{\mathrm{i}}$ \\
\hline 1 & 6 & 0.20094 & 9.4441 & 11 & 0.2563 \\
2 & 12 & 0.14658 & 6.8892 & 7 & 0.0018 \\
3 & 18 & 0.11571 & 5.4384 & 6 & 0.0580 \\
4 & 30 & 0.16883 & 7.9350 & 3 & 3.0692 \\
5 & 42 & 0.11295 & 5.3088 & 7 & 0.5388 \\
6 & 66 & 0.12996 & 6.1082 & 7 & 0.1302 \\
7 & $\infty$ & 0.12503 & 5.8763 & 6 & 0.0026 \\
& Totals & 1 & 47 & 47 & 4.0569 \\
\hline
\end{tabular}




\section{RESULTS}

\subsection{Randomized Neighborhood Search (RNS)}

Randomized neighborhood search is a numerical optimization method whose objective functions may be discontinuous and non-differentiable. This optimization is also known as a direct-search or derivative-free method. Randomized neighborhood search operates by iterative random moving from the initial solution to a better solution. The RNS algorithm is as follows:

Step 1 : Start from the initial parameters $\alpha$ and $\beta$. Compute the chi-squared value.

Step 2 : Randomly change the value $\alpha$ to $\alpha^{\prime}$ and $\beta$ to $\beta^{\prime}$. We can do this by choosing a uniform variate $\mu$ from the interval $[0,1]$ and let:

$$
\begin{gathered}
\alpha^{\prime}=\alpha+2(0.5-u)(0.1998), \\
\beta^{\prime}=\beta+2(0.5-u)(4.995)
\end{gathered}
$$

Step 3 : Compute chi-squared value with $\alpha^{\prime}$ and $\beta^{\prime}$.

Step 4 : Compare the chi-squared values which were obtained from steps 1 and 3.

If the chi-squared value of step 3 is greater than or equal to that of step 1 , then repeat step 2 .

If not, we set $\alpha=\alpha^{\prime}, \beta=\beta^{\prime}$ and then go on to step 2 .

Step 5 : Repeat until a termination criterion is met (adequate fitness reached).

From Table 1, we compute the mean $(\mu)$ and variance $\left(\sigma^{2}\right)$ :

$$
\begin{aligned}
& \mu=31.055319 \\
& \sigma^{2}=1,120.3337743
\end{aligned}
$$

When we replace $\mu$ and $\sigma^{2}$ in (8) and then approximate $\alpha$ by bisection, we get $\alpha=0.9286$. The approximate value of $\beta=30.0055$ can be obtained from (7). These two parameters $\alpha$ and $\beta$ will be used as the initial parameters for the RNS algorithm. We iterated RNS 10,000 times and obtained the results shown in Table 4.

Table 5 shows the shape parameters $\alpha$, scale parameters $\beta$ and chi-squared value using different estimation methods.
Table 4. Parameters $\alpha, \beta$ and chi-squared value by RNS

\begin{tabular}{llll}
\hline Times & $\alpha$ & $\beta$ & Chi-Squared \\
\hline 1 & 0.9286000000 & 30.0055000000 & 4.0569000000 \\
2 & 0.8381502696 & 33.0173413017 & 3.2266108642 \\
3 & 0.8381502696 & 33.0173413017 & 3.2266108642 \\
4 & 0.8381502696 & 33.0173413017 & 3.2266108642 \\
5 & 0.8381502696 & 33.0173413017 & 3.2266108642 \\
6 & 0.7076414583 & 28.7762666642 & 2.6481293827 \\
7 & 0.7076414583 & 28.7762666642 & 2.6481293827 \\
8 & 0.7076414583 & 28.7762666642 & 2.6481293827 \\
9 & 0.7076414583 & 28.7762666642 & 2.6481293827 \\
10 & 0.7076414583 & 28.7762666642 & 2.6481293827 \\
20 & 0.7095244694 & 29.5717808657 & 2.6140305005 \\
30 & 0.7148708496 & 26.9714010325 & 2.5856511398 \\
40 & 0.7148708496 & 26.9714010325 & 2.5856511398 \\
50 & 0.7148708496 & 26.9714010325 & 2.5856511398 \\
60 & 0.7148708496 & 26.9714010325 & 2.5856511398 \\
70 & 0.7148708496 & 26.9714010325 & 2.5856511398 \\
80 & 0.7131632905 & 30.1643026571 & 2.5559654901 \\
90 & 0.7131632905 & 30.1643026571 & 2.5559654901 \\
100 & 0.7131632905 & 30.1643026571 & 2.5559654901 \\
200 & 0.7160097628 & 28.1949938030 & 2.4788283052 \\
300 & 0.7160097628 & 28.1949938030 & 2.4788283052 \\
400 & 0.7160097628 & 28.1949938030 & 2.4788283052 \\
500 & 0.7160097628 & 28.1949938030 & 2.4788283052 \\
600 & 0.7160097628 & 28.1949938030 & 2.4788283052 \\
700 & 0.7160097628 & 28.1949938030 & 2.4788283052 \\
800 & 0.7157118026 & 28.4146840369 & 2.4783086176 \\
900 & 0.7157118026 & 28.4146840369 & 2.4783086176 \\
1,000 & 0.7158868924 & 28.4191078089 & 2.4745204778 \\
2,000 & 0.7157825238 & 28.7701321511 & 2.4707423531 \\
3,000 & 0.7158410970 & 28.7428309644 & 2.4697671190 \\
4,000 & 0.7158324217 & 28.7679580993 & 2.4697088719 \\
5,000 & 0.7158182813 & 28.8071827518 & 2.4696879774 \\
6,000 & 0.7158147162 & 28.8246778976 & 2.4696432384 \\
7,000 & 0.7158161544 & 28.8206039617 & 2.4696395832 \\
8,000 & 0.7158168650 & 28.8183923908 & 2.4696393961 \\
9,000 & 0.7158170080 & 28.8179670891 & 2.4696392513 \\
10,000 & 0.7158169062 & 28.8182970081 & 2.4696391693 \\
\hline & & &
\end{tabular}

Table 5. Chi-squared value for various estimation methods

\begin{tabular}{lllll}
\hline Method & Type & $\alpha$ & $\beta$ & Chi-Squared \\
\hline 1 & MLE & 0.8633 & 28.8668 & 5.9412 \\
2 & MOM (CV) & 0.9286 & 30.0055 & 4.0569 \\
3 & MOM (Cran) & 0.9552 & 30.4239 & 4.4097 \\
4 & LSM_1 & 0.8580 & 28.6168 & 5.9758 \\
5 & LSM_2 & 0.7984 & 29.1888 & 3.4099 \\
6 & LSM_3 & 0.8310 & 28.8602 & 6.0731 \\
7 & LSM_4 & 0.8405 & 28.7721 & 6.0239 \\
8 & WLSM_1 & 0.7647 & 29.9050 & 3.7284 \\
9 & WLSM_2 & 0.7455 & 30.1924 & 3.4214 \\
10 & WLSM_3 & 0.7571 & 30.0176 & 3.8360 \\
11 & WLSM_4 & 0.7600 & 29.9750 & 3.7936 \\
12 & WLSM_5 & 0.7967 & 29.2036 & 3.4216 \\
13 & WLSM_6 & 0.7710 & 29.5150 & 3.6609 \\
14 & WLSM_7 & 0.7868 & 29.3166 & 3.4988 \\
15 & WLSM_8 & 0.7907 & 29.2713 & 3.4662 \\
16 & RNS & 0.7158 & 28.8183 & 2.4696 \\
\hline
\end{tabular}




\section{DISCUSSION}

We should apply the RNS to other distributions for parameter estimation. The RNS should be applied to a mixture models; it is using the MLE via the Expectations-Maximization (EM) algorithm (Sattayatham and Talangtam (2012) for detail). In the other, we should consider the data of truncated and/or censored data sets in further research.

\section{CONCLUSION}

In this study, we have used RNS to estimate the Weibull parameters for the claim severity of fire accidents that cost more than 20 million baht. Table 5 shows RNS has the smallest chi-squared value (i.e., chisquared value $=2.4696$ ). Therefore RNS givesa more accurate estimation of parameters than do MLE, MOM, LSM or WLSM.

\section{ACKNOWLEDGMENT}

This research was partially supported by the Centre of Excellence in Mathematics, the Commission of Higher Education (CHE), Sriayudthaya Road, Bangkok 10140, Thailand.

\section{REFERENCES}

Cran, G.W., 1988. Moment estimators for the 3parameter Weibull distribution. IEEE Trans. Reliab., 37: 360-363. DOI: 10.1109/24.9839

El-Mezouar, Z.C., 2010. Estimation the shape location and scale parameters of the Weibull distribution. RTA, 4: 36-40.

Sattayatham, P. and T. Talangtam, 2012. Fitting of finite mixture distributions to motor insurance claims. J. Math. Stat., 8: 49-56. DOI: 10.3844/jmssp.2012.49.56

Seyit, A.A. and D. Ali, 2009. A new method to estimate Weibull parameters for wind energy application. Energy Conver. Mange., 50: 1761-1766. DOI: 10.1016/j.enconman.2009.03.020

Wu, D, J. Zhou and Y. Li, 2006. Methods for estimating weibull parameters for brittle materials. J. Mater. Sci., 41: 5630-5638. DOI: 10.1007/s10853-0060344-9

Yeliz, M.K., K. Mehmet and O.H. Fatih, 2011. Comparison of six different parameter estimation methods in wind power applications. Sci. Res. Essays, 6: 6594-6604. DOI: 10.5897/SRE11.549 